\title{
Research on the Optimal Allocation of Tolerances of CNC Machine Tools Based on Small Deformation of Grinding Wheel
}

Jinwei Fan ( $\sim$ jwfan@bjut.edu.cn )

Beijing University of Technology https://orcid.org/0000-0002-7766-9234

\section{Bentian Xie}

Beijing University of Technology

Qinzhi Zhao

China National Machine Tool Quality Supervision Testing Center

Junjian Wang

China National Machine Tool Quality Supervision Testing Center

Original Article

Keywords: Five-axis machine tool , Tolerance parameters , Precision optimization design , Slight deformation

Posted Date: September 20th, 2021

DOI: https://doi.org/10.21203/rs.3.rs-903136/v1

License: (9) This work is licensed under a Creative Commons Attribution 4.0 International License. Read Full License 


\section{Research on the Optimal Allocation of Tolerances of CNC Machine Tools Based on Small Deformation of Grinding Wheel}

Jin-Wei Fan, born in 1965,Graduated from Tianjin University in 1996 with a Ph.D., director of the Institute of Precision CNC Machining Technology, Beijing University of Technology. His main research direction is CNC technology, precision machining and servo control technology, China.

Tel: +86 18813053935; E-mail: jwfan@bjut.edu.cn

Ben-Tian Xie, born in 1997, Graduate student from the Institute of Precision CNC Machining Technology, Beijing University of Technology, his main research direction is CNC technology, precision machining and servo control technology, China.

E-mail:1540397074@qq.com

Qin-Zhi Zhaog, born in 1963, China National Machine Tool Quality Supervision Testing Center, China.

E-mail:zqz629@126.com

Jun-Jian Wang, born in 1965, China National Machine Tool Quality Supervision Testing Center, China.

E-mail: wangjunjian@jcs.gt.cn

Corresponding author: Jin-Wei Fan E-mail: jwfan@bjut.edu.cn 


\title{
Research on the Optimal Allocation of Tolerances of CNC Machine Tools Based on Small Deformation of Grinding Wheel
}

\author{
Jin-Wei Fan ${ }^{1}$ Ben-Tian Xie ${ }^{1}$ • Qin-Zhi Wang ${ }^{2}$ Jun-Jian Wang ${ }^{2}$
}

\begin{abstract}
In order to solve the problem of precision optimization design of CNC machine tools, this paper proposes an optimized allocation method of machine tool tolerance parameters that takes into account the slight deformation of the machine tool. First, establish a tolerance-based geometric error prediction model, and establish a spatial motion error model based on the theory of multi-body systems(MBS); Homogeneously, perform finite element analysis(FEA) on the $\mathrm{CNC}$ internal cylindrical compound grinding machine to obtain the slight deformation of the machine tool, and apply the result to the optimal tolerance allocation In the constraint conditions, the final optimal allocation plan is obtained; Finally, the genetic algorithm is used to simulate and analyze the plan, and the optimal tolerance allocation result is obtained. The result shows that most of the tolerance parameters have been relaxed, which means that the machine tool's manufacturing cost. Through experiments, it is verified that the optimized machine tool machining accuracy pass rates are $98.5 \%, 98.25 \%$, and $97.85 \%$, respectively. Therefore, the optimal allocation method of tolerances that considers small deformations proposed in this paper is effective.
\end{abstract}

Keywords: Five-axis machine tool • Tolerance parameters • Precision optimization design • Slight deformation

\section{Introduction}

Numerical control technology and machine tools are the technology and equipment support of a country engaged in high-end manufacturing, and many cutting-edge industries need numerical control technology as the basic technology

Jin-Wei Fan

jwfan@bjut.edu.cn

1 Beijing Key Laboratory of Advanced Manufacturing Technology,Beijing University of Technology, Beijing 100124, China

2 China National Machine Tool Quality Supervision Testing Center, Beijing 100102, China
[1]. Five-axis machine tools(FAMT) have obvious advantages of high material removal rate, less assembly and high processing efficiency, and are widely applicable to the processing of complex surfaces [2]. Machining accuracy is one of the important indexes to measure the performance of CNC machine tools, which is directly related to the quality of product processing, production cycle, production cost and market competitiveness. In the development of CNC machine tools, it has been striving to pursue higher machining accuracy, cutting speed, production efficiency and reliability[3]. Because the machining accuracy of machine tools is determined by the precision of the machine components in coordination with each other, improving the accuracy of individual components may increase manufacturing costs rather than improving machining accuracy. Therefore, in the initial design stage of machine tools, how to meet the requirements of machining accuracy, at the same time, to obtain the appropriate machine tool accuracy design economically and effectively, has important theoretical and practical significance.

Many engineering practices show that excellent machine tool precision design scheme, not only requires the machine to achieve high operation accuracy, but also requires the machine manufacturing cost as low as possible; Conversely, a poor precision design leads to tight or loose tolerances, high manufacturing costs and low machine performance. Numerical control machine tool precision optimization design work not only to consider in order to reduce the manufacturing cost of economic processing accuracy brought by the impact of machine tool processing accuracy, but also to consider in order to obtain higher machine tool processing accuracy and improve the accuracy of one or several parts increased manufacturing cost, weighing gains and losses.

Throughout the existing research literature, scholars have 
carried out a lot of research work on the optimization design of CNC machine tool accuracy. Jayaprakash et al. [4] used BP neural network to fit the cost-tolerance model, combined tolerance design with finite element analysis, to ensure that the best tolerance value of each component remained within the tolerance range. Sivakumar et al. [5] proposed a multi-objective optimization method for tolerance parallel design based on elite non-dominated sorting genetic algorithm (NSGA-II) and multi-objective particle swarm optimization (MOPSO). Taking into account the product sequence, Geetha et al. [6] allocated part tolerances by minimizing manufacturing costs (tolerance and quality loss cost) and machine idle time costs, and use genetic algorithm (GA) to achieve optimal allocation of part tolerance parameters. Cai et al. [7] proposed a method for optimal allocation of geometric accuracy of parts considering both reliability and robustness. This method established a cost model that comprehensively considered the present value of manufacturing costs and expected quality loss, and minimized the possibility and cost of machine tool failure. As the objective function, with the reliability of the machine tool as the constraint condition, the basic parameters of the machine tool are optimized, and the ability to maintain machining accuracy under certain design requirements is improved. Zhao et al. [8] established a product manufacturing cost model including machining dimensional tolerances and non-geometric tolerances, taking product manufacturing cost minimization as the objective function, and product-level quality characteristic tolerances as constraints, and finally established a product tolerance system optimization model. Liu et al. [9]also considered several commonly used manufacturing cost functions, including exponential function, inverse power function and polynomial function. Then, the total cost minimization was used as the objective function, and the tolerance parameters and the design standards of processing errors were used as constraints. The establishment of tolerance optimization allocation model. Liu Haibo et al. [10]established a multi-objective tolerance optimization model with processing cost, quality loss cost and tolerance sensitivity as the objective function, and solved it with an improved particle swarm optimization algorithm to obtain a uniform Pareto frontier. Shi Xiangling and others [11] established a multi-objective optimization mathematical model of assembly tolerance based on product cost and quality loss, and used the non-dominated sorting genetic algorithm (NSGA-II) with elite strategy to restrict assembly function requirements and processing capabilities. Optimize the solution to obtain the Pareto optimal solution set. Zhang et al. [12]took the minimization of the production cost of CNC machine tools as the objective function, established a geometric error optimization design model, and used optimization algorithms to complete the optimal allocation of various geometric error parameters, thereby improving the reliability of the machining accuracy of the machine tool.

Throughout the above research methods, the precision optimization and distribution of CNC machine tools has made a certain development, but the realization of these methods has a strong dependence on the design experience and level of machine tool design engineers, and there are still some important issues to be solved.

(1) The existing optimal tolerance allocation methods for machine tools are all based on the assumption that all parts of the machine tool are rigid bodies, ignoring the slight deformation of the machine tool. In fact, due to the gravity effect, the machine tool will inevitably have small deformations, and the machining accuracy of the machine tool is generally in the micron level, so the impact of the small deformation on the machining accuracy and manufacturing cost cannot be ignored.

(2) Part of the tolerance optimization method is carried out under certain constraints (i.e., $0<\mathrm{t} \leqslant$ initial value). The given initial value is not necessarily the range of the optimal solution, and the obtained optimization result is not necessarily Optimal results.

In view of the above limitations, in this paper, a new tolerance optimization allocation method is proposed. It is planned to use truncated Fourier series to establish the mapping relationship between the tolerances and geometric errors of the key parts of the machine tool, and use MBS theory and homogeneous transformation matrix(HTM) to establish spatial motion error model. Taking into account the slight deformation caused by the influence of the gravity of the machine tool, the tolerance parameters of the machine tool are used as design variables, and the tolerance parameter values and the spatial motion error of the machine tool do not exceed the design requirements as constraints, Taking the maximum machining accuracy of the whole machine, that is, minimizing the machining error and maximizing the sum of the product of each tolerance parameter and its corresponding weighting factor as the objective function, establish a multi-objective optimization distribution model based on the tolerance parameters, so that the machining accuracy and the whole machine The manufacturing cost is optimal. The flow chart as shown in Figure 1. 


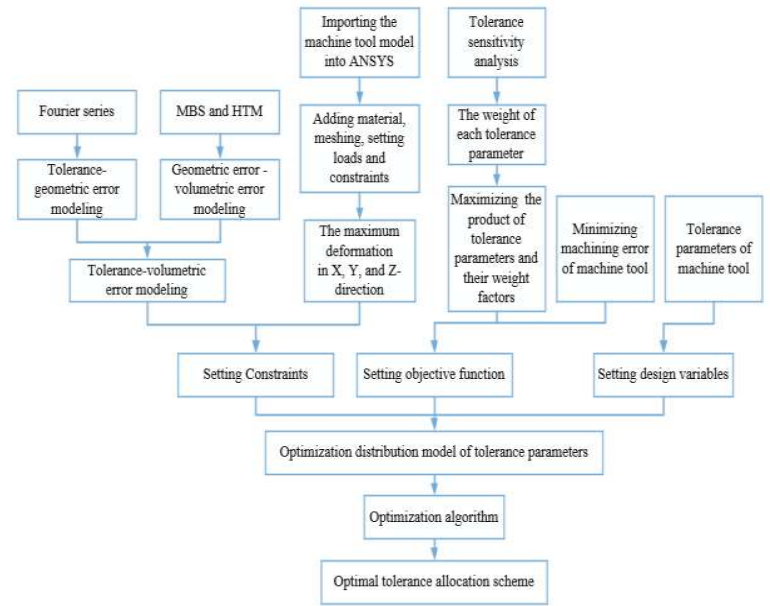

Figure 1 Flow chart of tolerance optimization allocation method

\section{Spatial motion error modeling of $\mathrm{CNC}$ machine tools based on tolerance}

Tolerance refers to the allowable variation of the size and geometric parameter values of product components, and is one of the important technical indicators in the process of product design, manufacturing, testing, assembly and testing [13]. In the precision design process of CNC machine tools, the geometric error of the machine tool plays a key role, but because the geometric error is generated after the machine tool is assembled, it is impossible to directly guide the precision design of the machine tool. Only the tolerance parameters of the key parts of the machine tool are known, so if the relationship model between the tolerances of the machine parts and the spatial motion error can be established, it can guide the subsequent optimization of the tolerance parameters of the machine tool.

\subsection{Geometric error modeling based on tolerance}

In this study, a CNC precision internal cylindrical compound grinder is taken as the research object. Its structure mainly includes bed, guide rail, headstock, workpiece, turret, cutting tool, etc., as shown in Figure 2. The main feature of this machine tool is the turret-type multi-spindle wheel box structure, which is equipped with three internal grinding wheels of different sizes and one external grinding wheel. The rotation of the turret realizes tool change to meet the needs of processing different types of shaft parts.

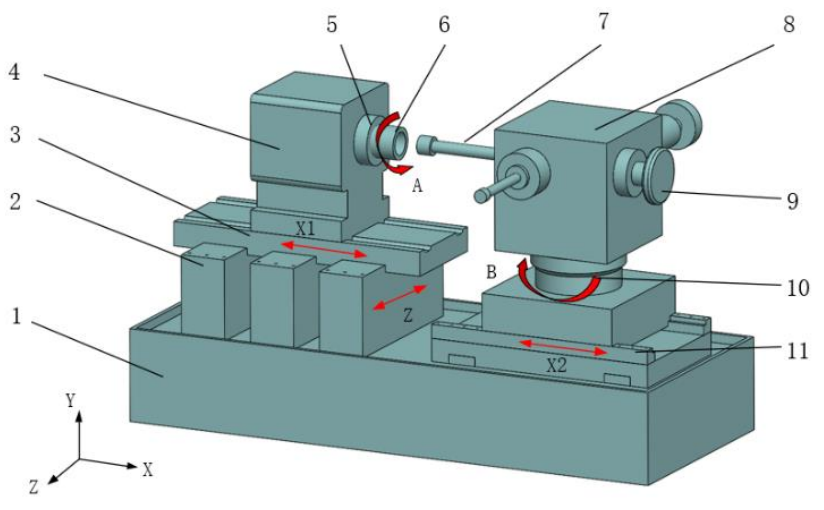

Figure 2 Schematic diagram of the structure of the $\mathrm{CNC}$ internal compound grinder

(1, machine bed; 2, Z-axis guide rail; 3, X1-axis guide rail; 4, headstock; 5 , headstock spindle; 6 , workpiece; 7 , Inner grinding wheel; 8 , grinding wheel box; 9 , external grinding wheel;10, turret; $11, \mathrm{X} 2$-axis guide rail)

Generally, the geometric errors of machine tools can be divided into position-related errors and position-independent errors [14]. The position-related error is the six-dimensional position and attitude error of each moving part during the movement of the machine tool, and the position-independent error is the position offset error of each part during the assembly process of the machine tool. According to the theory of space kinematics [15], each part of the machine tool has 6 degrees of freedom when moving in the coordinate system, and each degree of freedom corresponds to a position-related geometric error. In addition, it also involves the geometric error that has nothing to do with the position, i.e. the squareness error. All 36 geometric errors and the corresponding tolerance parameters involved in this machine tool are shown in Table 1, and the definitions of all tolerance parameters are shown in Table 2.

Table 1 Geometric errors and corresponding tolerance parameters

\begin{tabular}{cccccc}
\hline $\begin{array}{c}\text { Geometri } \\
\text { c error }\end{array}$ & $\begin{array}{c}\text { Toleranc } \\
\text { e }\end{array}$ & $\begin{array}{c}\text { Geometri } \\
\text { c error }\end{array}$ & $\begin{array}{c}\text { Toleranc } \\
\text { e }\end{array}$ & $\begin{array}{c}\text { Geometri } \\
\text { c error }\end{array}$ & $\begin{array}{c}\text { Toleranc } \\
\text { e }\end{array}$ \\
\hline$\delta_{x}\left(x_{1}\right)$ & $\mathrm{T} 1$ & $\delta_{x}(z)$ & $\mathrm{T} 9$ & $\delta_{x}(B)$ & $\mathrm{T} 16$ \\
$\delta_{y}\left(x_{1}\right)$ & $\mathrm{T} 2$ & $\delta_{y}(z)$ & $\mathrm{T} 10$ & $\delta_{y}(B)$ & $\mathrm{T} 17$ \\
$\delta_{z}\left(x_{1}\right)$ & $\mathrm{T} 3$ & $\delta_{z}(z)$ & $\mathrm{T} 11$ & $\delta_{z}(B)$ & $\mathrm{T} 16$ \\
$\varepsilon_{x}\left(x_{1}\right)$ & $\mathrm{T} 4$ & $\varepsilon_{x}(z)$ & $\mathrm{T} 10$ & $\varepsilon_{x}(B)$ & $\mathrm{T} 16$ \\
$\varepsilon_{y}\left(x_{1}\right)$ & $\mathrm{T} 3$ & $\varepsilon_{y}(z)$ & $\mathrm{T} 9$ & $\varepsilon_{y}(B)$ & $\mathrm{T} 18$ \\
$\varepsilon_{z}\left(x_{1}\right)$ & $\mathrm{T} 2$ & $\varepsilon_{z}(z)$ & $\mathrm{T} 12$ & $\varepsilon_{z}(B)$ & $\mathrm{T} 16$
\end{tabular}




\begin{tabular}{llllll}
$\delta_{x}\left(x_{2}\right)$ & $\mathrm{T} 5$ & $\delta_{x}(A)$ & $\mathrm{T} 14$ & $\varepsilon_{x_{1} B}$ & $\mathrm{~T} 19$ \\
$\delta_{y}\left(x_{2}\right)$ & $\mathrm{T} 6$ & $\delta_{y}(A)$ & $\mathrm{T} 13$ & $\varepsilon_{x_{2} B}$ & $\mathrm{~T} 20$ \\
$\delta_{z}\left(x_{2}\right)$ & $\mathrm{T} 7$ & $\delta_{z}(A)$ & $\mathrm{T} 13$ & $\varepsilon_{z A}$ & $\mathrm{~T} 21$ \\
$\varepsilon_{x}\left(x_{2}\right)$ & $\mathrm{T} 8$ & $\varepsilon_{x}(A)$ & $\mathrm{T} 15$ & $\varepsilon_{z B}$ & $\mathrm{~T} 22$ \\
$\varepsilon_{y}\left(x_{2}\right)$ & $\mathrm{T} 7$ & $\varepsilon_{y}(A)$ & $\mathrm{T} 13$ & $\varepsilon_{x_{1} z}$ & $\mathrm{~T} 23$ \\
$\varepsilon_{z}\left(x_{2}\right)$ & $\mathrm{T} 6$ & $\varepsilon_{z}(A)$ & $\mathrm{T} 13$ & $\varepsilon_{x_{2} z}$ & $\mathrm{~T} 24$ \\
\hline
\end{tabular}

Table 2 Definition of tolerance parameters

\begin{tabular}{|c|c|}
\hline $\begin{array}{l}\text { Tolerance } \\
\text { parameter }\end{array}$ & Definition of parameters \\
\hline T1 & The positioning tolerance of guide screw for X1-axis \\
\hline $\mathrm{T} 2$ & $\begin{array}{l}\text { The straightness tolerance of the guide rail of X1-axis in the } \\
X \text {-Y plane }\end{array}$ \\
\hline $\mathrm{T} 3$ & $\begin{array}{l}\text { The straightness tolerance of the guide rail of } \mathrm{X} 1 \text {-axis in the } \\
\mathrm{X}-\mathrm{Z} \text { plane }\end{array}$ \\
\hline T4 & Tolerance of parallelism between X1- axis guide pairs \\
\hline T5 & The positioning tolerance of guide screw for X2-axis \\
\hline T6 & $\begin{array}{l}\text { The straightness tolerance of the guide rail of } \mathrm{X} 2 \text {-axis in the } \\
\mathrm{X} \text {-Y plane }\end{array}$ \\
\hline $\mathrm{T} 7$ & $\begin{array}{l}\text { The straightness tolerance of the guide rail of } \mathrm{X} 2 \text {-axis in the } \\
\mathrm{X}-\mathrm{Z} \text { plane }\end{array}$ \\
\hline $\mathrm{T} 8$ & Tolerance of parallelism between $\mathrm{X} 2$ - axis guide pairs \\
\hline T9 & $\begin{array}{l}\text { The straightness tolerance of the guide rail of } Z \text {-axis in the } \\
X-Z \text { plane }\end{array}$ \\
\hline T10 & $\begin{array}{l}\text { The straightness tolerance of the guide rail of Z-axis in the } \\
\text { Y-Z plane }\end{array}$ \\
\hline T11 & The positioning tolerance of guide screw for $\mathrm{Z}$-axis \\
\hline T12 & Tolerance of parallelism between Z- axis guide pairs \\
\hline T13 & The radial runout tolerance of the A-axis \\
\hline T14 & The axial runout tolerance of the A-axis \\
\hline T15 & The positioning tolerance of the A-axis \\
\hline T16 & The radial runout tolerance of the $\mathrm{B}$-axis \\
\hline T17 & The axial runout tolerance of the B-axis \\
\hline T18 & The positioning tolerance of the B-axis \\
\hline T19 & The perpendicularity tolerance between $\mathrm{X} 1$-axis and $\mathrm{B}$-axis \\
\hline $\mathrm{T} 20$ & The perpendicularity tolerance between $\mathrm{X} 2$-axis and $\mathrm{B}$-axis \\
\hline $\mathrm{T} 21$ & The perpendicularity tolerance between $\mathrm{Z}$-axis and A-axis \\
\hline T22 & The perpendicularity tolerance between $\mathrm{Z}$-axis and $\mathrm{B}$-axis \\
\hline $\mathrm{T} 23$ & The perpendicularity tolerance between $\mathrm{X} 1$-axis and Z-axis \\
\hline $\mathrm{T} 24$ & The perpendicularity tolerance between $\mathrm{X} 2$-axis and $\mathrm{Z}$-axis \\
\hline
\end{tabular}

In this paper, the surface micro-morphology of the key parts of CNC machine tools and the assembly relationship between the parts are used as a bridge between tolerances and geometric errors, and a geometric error prediction model based on the tolerances of key parts of CNC machine tools is established. According to the Dirichlet boundary conditions [16], the surface error morphology of parts can be characterized by Fourier series, and the first term of the Fourier series which has a significant influence on the motion error is selected to characterize the surface error morphology of the parts. Firstly, establish the relationship model between the tolerance and the surface error morphology of the machine tool's translational axis and the rotary axis. According to the assembly relationship between the various parts of the machine tool, establish the machine tool's translational axis and the rotary axis's surface error morphology and various geometric errors. The relationship model of, and then the mapping relationship between tolerance and geometric error can be derived.
Due to limited space, only the results of the geometric error model for a linear axis and a rotary axis (i.e., $\mathrm{X}$-axis and B-axis) are given, as shown in Appendix 1. More details of the modeling process can refer to the previous work[17].

\subsection{Spatial motion error modeling}

In this study, the spatial motion error modeling of the grinder is analyzed and studied based on the MBS theory [18]. First, the mechanical structure is abstracted into a simple body form through the topological structure. According to the structure of the grinder, it can be divided into the workpiece branch and the grinding wheel. Branch, where the workpiece branches into the bed (body 0), Z-direction guide (body 1), X1-direction guide (body 2), headstock spindle (body 3) and workpiece (W); grinding wheel branches into the bed, X2-direction Guide rail (body 4), turret (body 5), grinding wheel box (body 6) and grinding wheel $(\mathrm{T})$, the kinematic chain structure is shown in Figure 3.

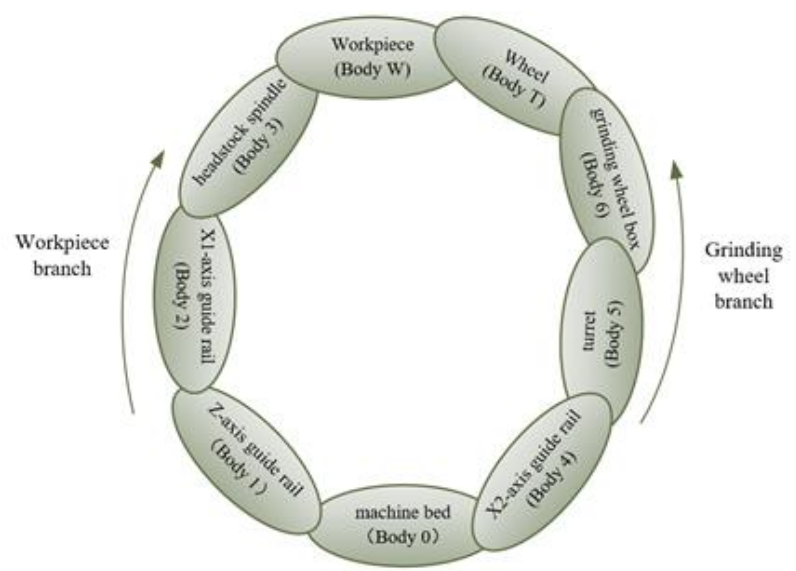

Figure 3 Kinematic chain structure diagram

According to the principle of homogeneous coordinate transformation, set $\mathrm{E}$ as the unit matrix, and establish the ideal transformation matrix $[S I J]_{p}$, relative position error transformation matrix $[S I J]_{p e}$, relative motion ideal transformation matrix $[S I J]_{S}$ and relative motion error transformation matrix $[S I J]_{s e}$ of the workpiece motion chain branch and the grinding wheel motion chain branch.where, $I, J=0,1, \mathrm{~K}, 6$.

$\mathrm{P}$ is Supposed as a grinding point of the grinding wheel, the homogeneous coordinate of point $\mathrm{P}$ in the grinding wheel coordinate system is $\boldsymbol{r}_{T}=\left(\begin{array}{lllll}0 & 0 & -R & 1\end{array}\right)^{T}$, and the homogeneous coordinate of point $\mathrm{P}$ in the workpiece coordinate system is $\boldsymbol{r}_{w}=\left(\begin{array}{llll}r_{w x} & r_{w y} & r_{w z} & 1\end{array}\right)^{T}$, where $\mathrm{R}$ is the radius of the grinding wheel. In the actual working condition of the machine tool, the position vector 
expression of the grinding point $\mathrm{P}$ along the workpiece branch in the machine tool coordinate system, as shown in Eq.(1):

$$
\begin{aligned}
\boldsymbol{P}_{w}= & \prod_{t=n, L^{n}(j)=1}^{t=1}\left(\left[S L^{t}(j) L^{t-1}(j)\right]_{p}\left[S L^{t}(j) L^{t-1}(j)\right]_{p e}\right)\left\{r_{w}\right\}_{w} \\
= & {[S 01]_{p}[S 01]_{p e}[S 01]_{s}[S 01]_{s e}[S 12]_{p}[S 12]_{p e} } \\
& {[S 12]_{s}[S 12]_{s e}[S 23]_{p}[S 23]_{p e}[S 23]_{s}[S 23]_{s e} } \\
& {[S 3 W]_{p}[S 3 W]_{p e}[S 3 W]_{s}[S 3 W]_{s e} \boldsymbol{r}_{w} }
\end{aligned}
$$

The position vector expression of the grinding point $\mathrm{P}$ along the grinding wheel branch in the machine tool coordinate system, as shown in Eq.(2):

$$
\begin{aligned}
\boldsymbol{P}_{T}= & \prod_{t=n, L^{n}(j)=1}^{t=1}\left(\left[S L^{t}(j) L^{t-1}(j)\right]_{p}\left[S L^{t}(j) L^{t-1}(j)\right]_{p e}\right. \\
= & {[S 04]_{p}[S 04]_{p e}[S 04]_{s}[S 04]_{s e}[S 45]_{p}[S 45]_{p e} } \\
& {[S 45]_{s}[S 45]_{s e}[S 56]_{p}[S 56]_{p e}[S 56]_{s}[S 56]_{s e} } \\
& {[S 6 T]_{p}[S 6 T]_{p e}[S 6 T]_{s}[S 6 T]_{s e} \boldsymbol{r}_{T} }
\end{aligned}
$$

To achieve the precision machining requirements of the grinder, it is necessary to meet the coincidence of the grinding point in the workpiece branch and the grinding point in the grinding wheel branch, , as shown in Eq.(3):

$$
\boldsymbol{P}_{w}=\boldsymbol{P}_{T}
$$

In summary, the position vector of the grinding point of the grinding wheel in the workpiece coordinate system in the actual machining process, as shown in Eq.(4):

$$
\begin{aligned}
\boldsymbol{r}_{w}= & {[S 01]_{p}[S 01]_{p e}[S 01]_{s}[S 01]_{s e}[S 12]_{p}[S 12]_{p e} } \\
& {[S 12]_{s}[S 12]_{s e}[S 23]_{p}[S 23]_{p e}[S 23]_{s}[S 23]_{s e} } \\
& {\left.[S 3 W]_{p}[S 3 W]_{p e}[S 3 W]_{s}[S 3 W]_{s e}\right)^{-1}[S 04]_{p} } \\
& {[S 04]_{p e}[S 04]_{s}[S 04]_{s e}[S 45]_{p}[S 45]_{p e}[S 45]_{s} } \\
& {[S 45]_{s e}[S 56]_{p}[S 56]_{p e}[S 56]_{s}[S 56]_{s e}[S 6 T]_{p} } \\
& {[S 6 T]_{p e}[S 6 T]_{s}[S 6 T]_{s e} \boldsymbol{r}_{T} }
\end{aligned}
$$

Substituting the established homogeneous transformation matrix into the above formula, the actual grinding wheel grinding point position $\boldsymbol{r}_{w}$ is obtained, and the error matrix is eliminated to obtain the ideal grinding wheel grinding point position $\boldsymbol{r}_{w}{ }^{i}$. Subtract the two to get the spatial motion error model of $\mathrm{CNC}$ internal cylindrical compound grinder, as shown in Eq.(5):

$$
\boldsymbol{E}=\boldsymbol{r}_{w}-\boldsymbol{r}_{w}{ }^{i}=\left(\begin{array}{llll}
E_{x} & E_{y} & E_{z} & 0
\end{array}\right)^{T}
$$

Where, $E_{x}$ represents the component of the spatial motion error of the whole machine in the X direction; $E_{y}$ represents the component of the whole machine's spatial motion error in the $\mathrm{Y}$ direction; $E_{z}$ represents the component of the whole machine's spatial motion error in the $\mathrm{Z}$ direction. More details are shown in Appendix 2.

Solving the matrix with MATLAB, sorting out the results and eliminating high-order terms, can get a complete grinder spatial motion error model, and then according to the tolerance and geometric error model established in Section 2.1, to obtain a tolerance-based spatial motion error model.

\section{Tolerance optimization distribution of CNC internal cylindrical compound grinder}

The manufacturing cost of a machine tool is directly related to the tolerances of its parts. If the accuracy of the parts is blindly improved, it is easy to cause the problems of high manufacturing costs and low machining accuracy of the whole machine. The purpose of the optimal allocation of tolerance parameters is to maximize the control of the tolerance parameters of machine tool parts under the premise of ensuring product performance, that is, to minimize the manufacturing cost of the machine tool. Therefore, it is of great practical significance to seek a reasonable and effective method of optimal allocation of tolerance parameters.

In this chapter, the 24 tolerance parameters involved in the $\mathrm{CNC}$ internal compound grinding machine are used as design variables, and the tolerance parameters and the machine's spatial motion error meet the accuracy requirements as the constraints, and the overall machining error is minimized and the tolerance parameter values The maximum sum of the product of the corresponding weighting factors (sensitivity coefficient) is the objective function, the multi-objective optimization allocation model of tolerance parameters is established, and the NSGA-II is used to solve the model, thereby completing the numerical control inner circle compound The tolerance parameters of the grinder are optimally allocated.

\subsection{Sensitivity analysis model}

Sensitivity analysis is a method to study the sensitivity of a system's state and output changes to system parameters or 
changes in surrounding conditions. The sensitivity analysis of a machine tool can obtain the degree of influence of tolerance parameters on machining accuracy, and then guide the subsequent optimization of machine tool tolerance allocation.

Based on the established relationship model between the tolerance parameters and geometric errors of the key parts of CNC internal cylindrical compound grinders, this paper regards the machining errors caused by each geometric error alone as the sensitivity corresponding to each geometric error, and each geometric error is taken as The intermediate variable obtains the sensitivity of the tolerance parameters of the key parts of the machine tool to the spatial operation accuracy of the whole machine, as shown in Eq.(6) and Eq.(7):

$$
\begin{gathered}
\Delta E_{T}=\left[\begin{array}{c}
\Delta E_{T x} \\
\Delta E_{T y} \\
\Delta E_{T y} \\
0
\end{array}\right]=\frac{\partial E}{\partial G n} \cdot \frac{d G n}{d T n} \\
S_{T}=\sqrt{\Delta{E_{T x}}^{2}+\Delta E_{T y}{ }^{2}+\Delta E_{T z}{ }^{2}}
\end{gathered}
$$

Where, represents the nth geometric error of the machine tool; represents the tolerance parameter related to the nth geometric error; represents the increment of the tolerance parameter $\mathrm{T}$ to the spatial motion error of the machine tool; represents the sensitivity of tolerance parameter $\mathrm{T}$ to spatial motion error.

In order to more intuitively identify the key geometric errors and assign weights to each geometric error, the sensitivity of each tolerance is normalized, and the sensitivity coefficient Sn of each tolerance can be obtained, as shown in Eq.(8):

$$
S n=\frac{\left|S_{T}\right|}{\sum\left|S_{T}\right|}
$$

According to the previous research, it can be known that a tolerance parameter can correspond to multiple geometric errors. In this paper, the maximum value of multiple geometric error sensitivity coefficients is taken as the sensitivity coefficient of this tolerance parameter. Therefore, the sensitivity coefficients of various tolerance parameters can be obtained, as shown in Figure 4. The key tolerance parameter items can be directly obtained from the figure, which lays the foundation for the optimization of the next tolerance parameter allocation.

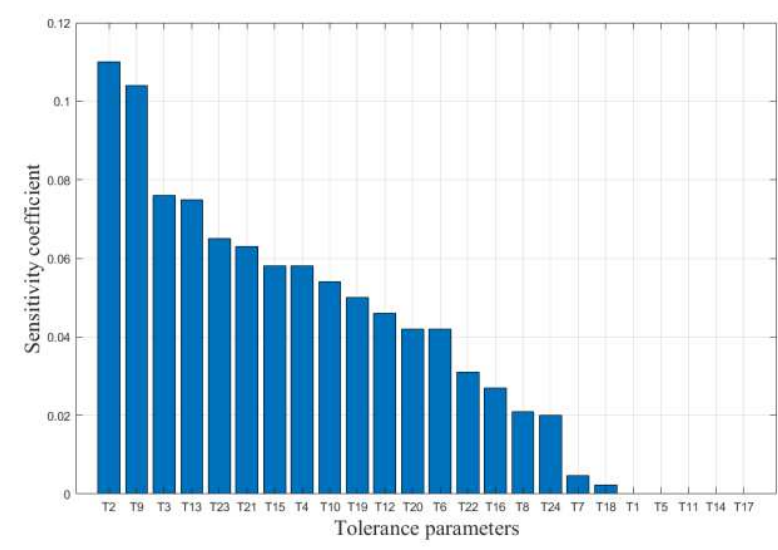

Figure 4 Sensitivity coefficients of various tolerance parameters

\subsection{Optimal allocation model of tolerance parameters}

\subsubsection{Determination of design variables}

In this study, the 24 tolerance parameters mentioned in section 2.1 are selected as the design variables, as shown in Eq.(9):

$$
T n=\left\{\begin{array}{llll}
T 1 & T 2 & \ldots & T 24
\end{array}\right\}
$$

Where, T1, T2,.., T24 are the tolerance parameters involved in the grinder.

\subsubsection{Determination of constraint conditions}

Constraint condition is a kind of restriction condition for the value range of design variables. According to the precision design requirements of $\mathrm{CNC}$ internal cylindrical compound grinding machine, the constraint conditions of the tolerance parameter optimization allocation model proposed in this paper are mainly divided into the whole machine space motion error constraint and various Tolerance parameter constraints.

At present, the research work on machine tool spatial motion error modeling is mainly carried out based on multi-body system theory. The prerequisite of multi-body system theory is to assume that all parts of CNC machine tools are rigid bodies. However, in the actual production and processing process, due to its own gravity As well as the effect of cutting force, the parts of CNC machine tools will inevitably produce small deformations, which will cause the position of the tool center point to shift, and the machining accuracy of the machine tool is generally in the micron level. Therefore, this position shift has an indispensable effect on the machining accuracy. Ignore the impact. In order to solve the problem of ignoring the position offset of the tool center point, this paper uses ANSYS Workbench 2020R1 to analyze the offset of the 
center point of the grinding wheel due to its own gravity and cutting force, and takes it into account in the final spatial motion error. The finite element analysis process can be divided into the following five steps:

(1) Establishment of three-dimensional model of machine tool

This paper uses SolidWorks 2019 to establish a three-dimensional model of a $\mathrm{CNC}$ internal cylindrical compound grinder, and reasonably simplifies the constructed geometric model during the three-dimensional modeling process, and removes the fine process features;

(2) Define the material properties of each key component of the machine tool

When performing statics analysis on CNC internal compound grinding machines, it is necessary to define the elastic modulus, Poisson's ratio, density, etc. of each key component material in advance;

(3) Meshing

The quality of the meshing directly has an important impact on the calculation time and calculation accuracy of the finite element analysis

(4) Add loads and constraints

First, set the fixed constraint on the machine bed, set the cylindrical support constraint boundary conditions in the installation bores of the A and B axes, and set the displacement constraint boundary conditions on the $\mathrm{X} 1$, $\mathrm{X} 2$, and $\mathrm{Z}$ axes. Then, in order to consider the load effects such as gravity and cutting force, A gravity load is applied to the whole machine, and a cutting force load is applied to the tool;

(5) Analysis results

According to the above steps, the finite element analysis and modeling work of the $\mathrm{CNC}$ internal cylindrical compound grinding machine is completed, and the offset of the center point of the grinding wheel in the $\mathrm{X}, \mathrm{Y}$ and $\mathrm{Z}$ directions is obtained. The deformation cloud diagram is shown in Figure 5, and the deformation amounts are $l_{x}=0.0016 \mathrm{~mm}, l_{y}=0.01 \mathrm{~mm}$, $l_{z}=0.0007 \mathrm{~mm}$.

According to the design criteria of the spatial operation accuracy of this grinder, the spatial movement error of the grinding wheel in the $\mathrm{X}, \mathrm{Y}$ and $\mathrm{Z}$ directions cannot exceed $0.02 \mathrm{~mm}$. In order to meet the design standards of the grinder, when optimizing the design, it is necessary to ensure that the components of the spatial motion error of the grinder in the $\mathrm{X}, \mathrm{Y}$, and $\mathrm{Z}$ directions meet the following requirements:

$$
\left\{\begin{array}{c}
E_{x}+0.0016 \leq 0.02 \\
E_{y}+0.01 \leq 0.02 \\
E_{z}+0.0007 \leq 0.02
\end{array}\right.
$$

In the actual manufacturing process of the grinder, it is impossible for the tolerance parameter value of each key component to reach the ideal state, i.e. $T_{n}=0$, so the tolerance parameter value is set to $T_{n}>0$. According to the accuracy inspection standard of $\mathrm{CNC}$ internal compound grinder, the given standard value of the tolerance parameter of each key component can be obtained. The tolerance parameter optimization allocation method proposed in this paper is to make each tolerance parameter Maximize the feasible range. If the maximum value of each tolerance parameter is set to infinite, it does not conform to the actual project. Therefore, the maximum value of the tolerance zone width is appropriately enlarged. This stduy uses 2 times the standard value of each tolerance parameter as its maximum value, and each can be obtained. The constraints of tolerance parameters are:

$$
0<\operatorname{Tn}<2 \operatorname{Tn} b
$$

Where, Tnb represents the value of the nth tolerance parameter given by the $\mathrm{CNC}$ internal cylindrical compound grinder standard.

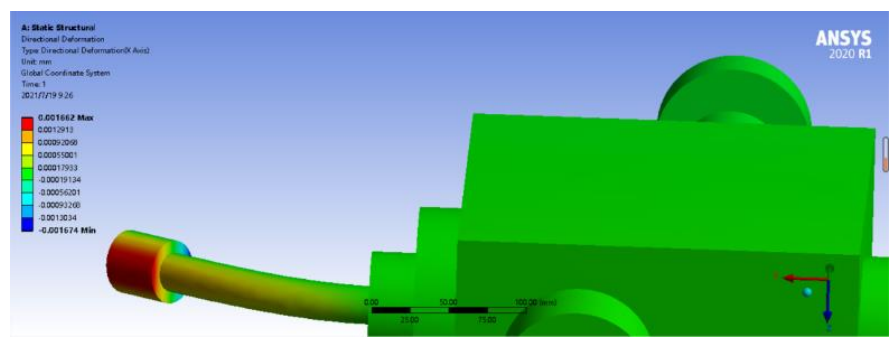

(a) $\mathrm{X}$-axis

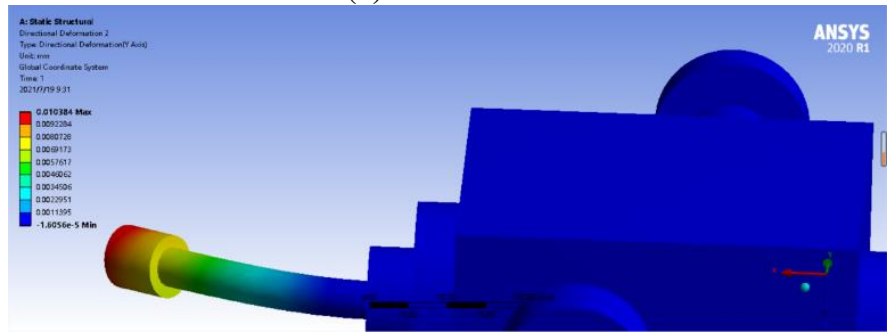

(b) Y-axis

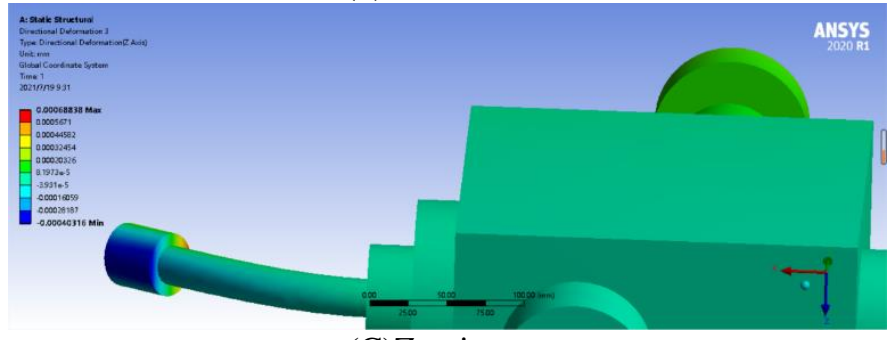

(C)Z-axis

Figure 5 Deformation cloud diagram of the center of the grinding wheel

\subsubsection{Determination of the objective function}

Most engineering design problems have multiple goals 
and need to be met at the same time, that is, there are multiple nonlinear goals in the same problem model at the same time, and these objective functions need to be optimized at the same time, and they often conflict with each other. This kind of optimization problem with multiple objective functions under given constraints is called a multi-objective optimization problem. The multi-objective optimization process considers the coupling relationship between the various objective functions, can reasonably balance the requirements of each objective function, and obtain a set of optimal solution sets at the same time, providing design engineers with multiple reasonable design options to choose from.

In the existing research, the tolerance-cost model plays an important role in the optimal allocation of tolerance parameters of the machine tool, but its applicability and reliability are not strong from the perspective of practical application, because the processing of each key component of the machine tool The relationship function between cost and tolerance is obtained by collating and summarizing the mass production experience data collected by experts, scholars and enterprise design engineers, and there are many factors affecting the processing cost of each component, such as processing technology, manufacturer's experience, processing Processes, production technologies, and different manufacturing companies will have different processing cost experience data for the same component, so different tolerance-cost relationship curves will be generated $[19,20]$. By studying various commonly used tolerance-cost models, it can be found that although the tolerance-cost relationship curve is different, there is a certain inverse proportional relationship between the two, that is, the larger the tolerance parameter value, the lower the cost. In order to solve the problem of low reliability and applicability of the tolerance-cost model mentioned above, this paper takes the maximization of the sum of the product of each tolerance parameter and its corresponding weight factor and the minimization of the machining error of the whole machine as the design goals, and establishes the tolerance parameter optimization allocation model.

The maximum value of the sum of the product of each tolerance parameter and its corresponding weighting factor are defined as the objective function 1, as shown in Eq.(12):

$$
\max f(T)=\sum_{n=1}^{24} S n \cdot T n
$$

Where, $S n$ represents the sensitivity coefficient of the nth tolerance parameter, $T n$ represents the value of the nth tolerance parameter.

When solving the optimal allocation model, the minimum value of the objective function is required, as shown in Eq.(13):

$$
\min f_{1}(T)=-\sum_{n=1}^{24} S n \cdot T n
$$

The minimization of the spatial motion error of the whole grinder is defined as the objective function 2 , as shown in Eq.(14):

$$
\min f_{2}(T)=\sqrt{E_{x}^{2}+E_{y}^{2}+E_{z}^{2}}
$$

In summary, the multi-objective optimization design function of the tolerance parameters of the key components of the $\mathrm{CNC}$ internal cylindrical compound grinder can be obtained, as shown in Eq.(15):

$$
\min \quad F(T)=\left(f_{1}(T), f_{2}(T)\right)
$$

\subsubsection{Determination of the optimal allocation model of tolerance parameters}

Based on the above formula, the standard expression form of the tolerance parameter optimization allocation model can be obtained as:

$$
\left\{\begin{array}{cc}
\min & F(T)=\left(f_{1}(T), f_{2}(T)\right) \\
\text { s.t. } & E_{x}+0.0016 \leq 0.02 \\
& E_{y}+0.01 \leq 0.02 \\
& E_{z}+0.0007 \leq 0.02 \\
& 0<T n<2 T n b \\
& n=1,2,3, \ldots, 24
\end{array}\right.
$$

\subsection{Simulation verification}

\subsubsection{Optimization algorithm}

NSGA-II algorithm (non-dominant sorting genetic algorithm with elite strategy) is an algorithm that searches for the optimal solution by simulating Darwin's genetic selection and natural elimination biological evolution process [21]. The algorithm is simple, universal, and robust. It is highly flexible, suitable for parallel processing, and is widely used in various fields, especially for some classical application fields of nonlinear and multi-objective large-scale and complex mechanical system optimization design. NSGA-II algorithm is superior to other optimization algorithms, effectively reduces the risk of falling into local optimal solution in the optimization process, and can obtain the optimal solution more conveniently and accurately.

In order to verify the correctness of the proposed tolerance optimization distribution model for key parts of $\mathrm{CNC}$ internal cylindrical compound grinding machine, the tolerance optimization distribution model was solved based on the NSGA-II algorithm and MATLAB R2021a. The Figure 6 shows the flow chart of the optimal allocation of tolerance parameters. In the figure, M 
represents the population size, $G$ represents the number of iterations, $\eta_{1}$ represents the simulation binary crossover parameter, $\eta_{2}$ represents the polynomial mutation parameter, $P_{c}$ represents the crossover probability, $P_{v}$ represents the mutation probability, and $\mathrm{Sn}$ represents The sensitivity coefficient corresponding to each tolerance parameter, and the value of each key parameter are shown in Table 3.

Table 3 Parameters in the optimization model

\begin{tabular}{ll}
\hline Parameters & Value \\
\hline Population size & 300 \\
Crossover probability & 0.8 \\
Mutation probability & 0.05 \\
The number of iterations & 200 \\
Analog binary cross parameter & 2 \\
Polynomial variation parameter & 5 \\
\hline
\end{tabular}

Based on the NSGA-II algorithm and using MATLAB R2016b, the tolerance parameters of the key parts of the gantry five-axis CNC milling machine were optimized for multi-objective allocation, and finally the Pareto optimal solution set was obtained, as shown in Figure 7. Aiming at the specific example of the optimization design of the tolerance parameters of the key components of the CNC precision internal cylindrical compound grinding machine, the machine tool design engineer was discussed, and the final tolerance optimization distribution plan was determined from the Pareto optimal solution set according to the actual production conditions of the enterprise.Compare the tolerance parameter values before and after optimization. A positive difference between the two indicates that the tolerance parameter item has been relaxed. On the contrary, a negative difference between the two indicates that the tolerance parameter item has been tightened, as shown in Table 4.

Table 4 Comparison of tolerance parameters before and after optimization

\begin{tabular}{|c|c|c|c|}
\hline $\begin{array}{c}\text { Tolerance } \\
\text { parameters }\end{array}$ & $\begin{array}{c}\text { Before } \\
\text { optimization(mm) }\end{array}$ & $\begin{array}{c}\text { After } \\
\text { optimization(mm) }\end{array}$ & Difference(mm) \\
\hline T1 & 0.006 & 0.007 & 0.001 \\
\hline T2 & 0.01 & 0.005 & -0.005 \\
\hline T3 & 0.012 & 0.025 & 0.013 \\
\hline T4 & 0.015 & 0.023 & 0.008 \\
\hline T5 & 0.006 & 0.008 & 0.002 \\
\hline T6 & 0.01 & 0.015 & 0.005 \\
\hline T7 & 0.012 & 0.014 & 0.004 \\
\hline T8 & 0.015 & 0.019 & 0.003 \\
\hline T9 & 0.015 & 0.02 & 0.005 \\
\hline T10 & 0.008 & 0.01 & 0.002 \\
\hline T11 & 0.005 & 0.006 & 0.001 \\
\hline T12 & 0.02 & 0.031 & 0.011 \\
\hline T13 & 0.01 & 0.018 & 0.008 \\
\hline
\end{tabular}

\begin{tabular}{|c|c|c|c|}
\hline T14 & 0.008 & 0.0065 & -0.0015 \\
\hline T15 & $0.05 / 1000$ & $0.056 / 1000$ & $0.006 / 1000$ \\
\hline T16 & 0.01 & 0.009 & -0.001 \\
\hline T17 & 0.007 & 0.015 & 0.008 \\
\hline T18 & $0.04 / 1000$ & $0.016 / 1000$ & $-0.024 / 1000$ \\
\hline T19 & $0.03 / 500$ & $0.027 / 500$ & $-0.003 / 500$ \\
\hline T20 & $0.03 / 500$ & $0.027 / 500$ & $-0.003 / 500$ \\
\hline T21 & $0.02 / 500$ & $0.035 / 500$ & $0.015 / 500$ \\
\hline T22 & $0.025 / 500$ & $0.02 / 500$ & $-0.005 / 500$ \\
\hline T23 & $0.02 / 500$ & $0.035 / 500$ & $0.015 / 500$ \\
\hline T24 & $0.02 / 500$ & $0.035 / 500$ & $0.015 / 500$ \\
\hline
\end{tabular}

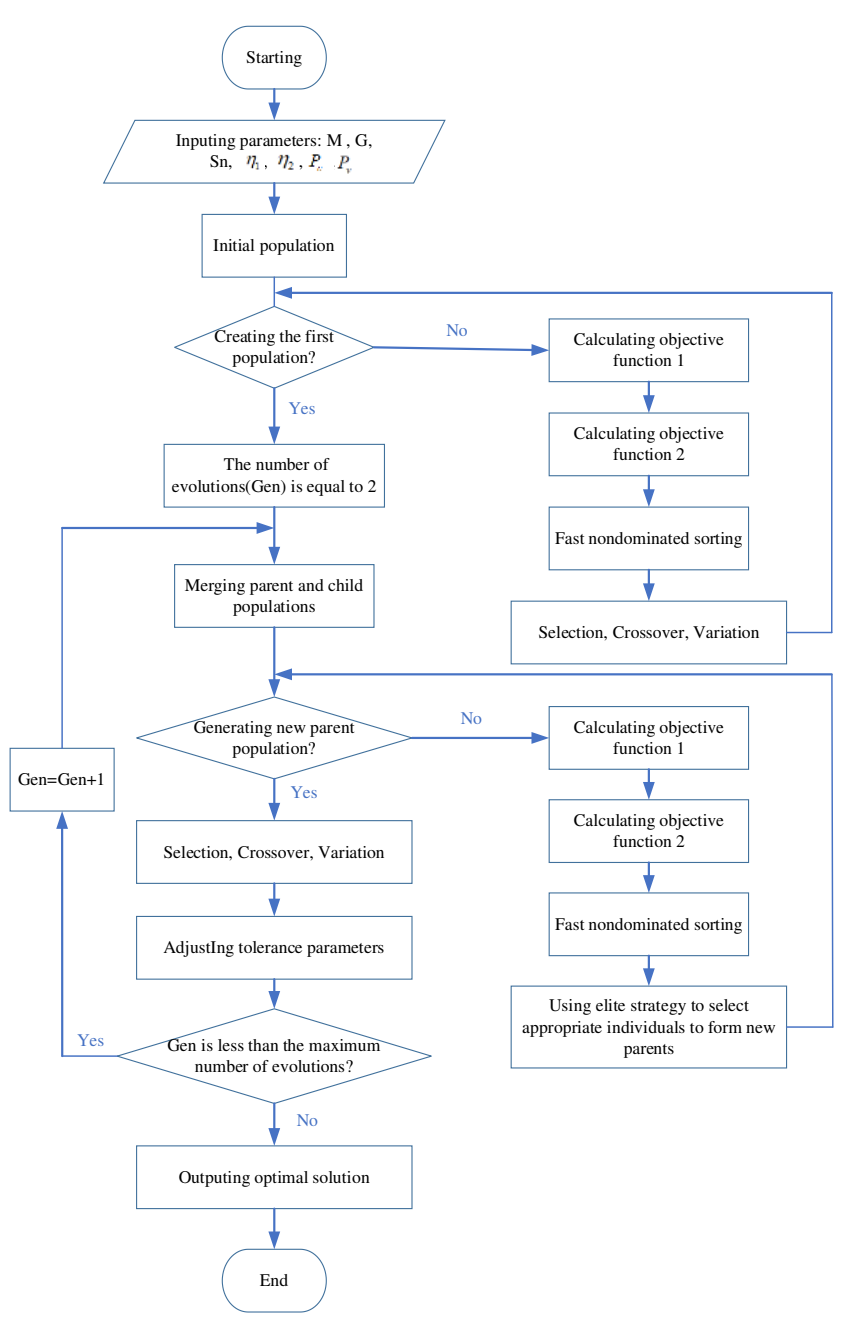

Figure 6 Flow chart of the optimal allocation of tolerance parameters 


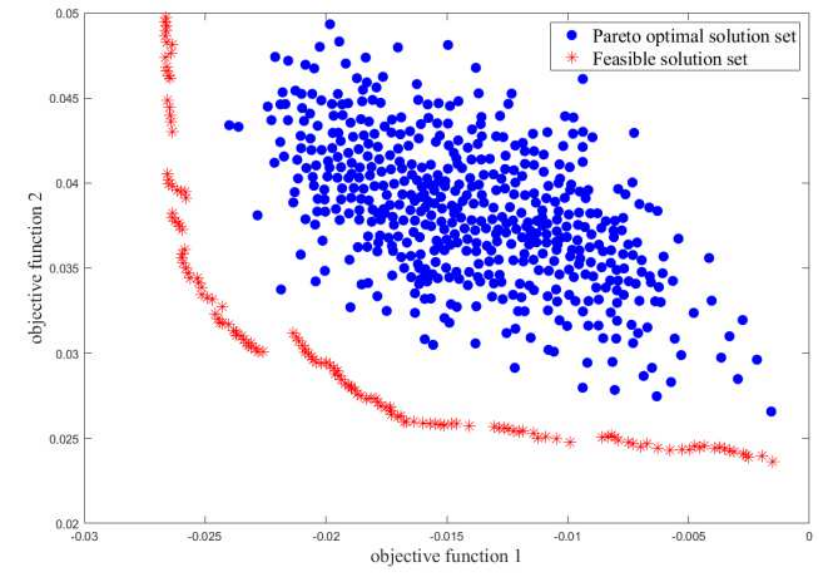

Figure 7 Tolerance optimization distribution plan of key components of $\mathrm{CNC}$ precision internal cylindrical compound grinder

In order to more intuitively reflect the advantages of the final tolerance optimization allocation plan, the histogram is used to compare the tolerance parameters before and after optimization. The tolerance parameters corresponding to linear errors are generally in millimeters, and the tolerance parameters corresponding to angular errors are generally in the form of ratios.

According to Figure 8, it can be seen intuitively that most of the tolerance parameter items have been relaxed to varying degrees, and the key tolerance parameters have also been relaxed to varying degrees. Because there is a certain inverse relationship between tolerance and cost, it is indirectly It shows that the manufacturing cost of the whole machine can be reduced. Therefore, the comparison results verify the feasibility and effectiveness of the optimal allocation method for the tolerance parameters of the key parts of the $\mathrm{CNC}$ internal cylindrical compound grinding machine proposed in this paper.

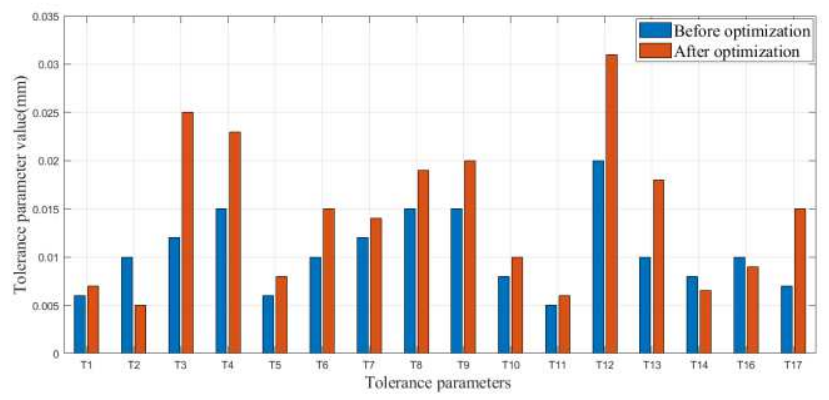

(a) Linear error

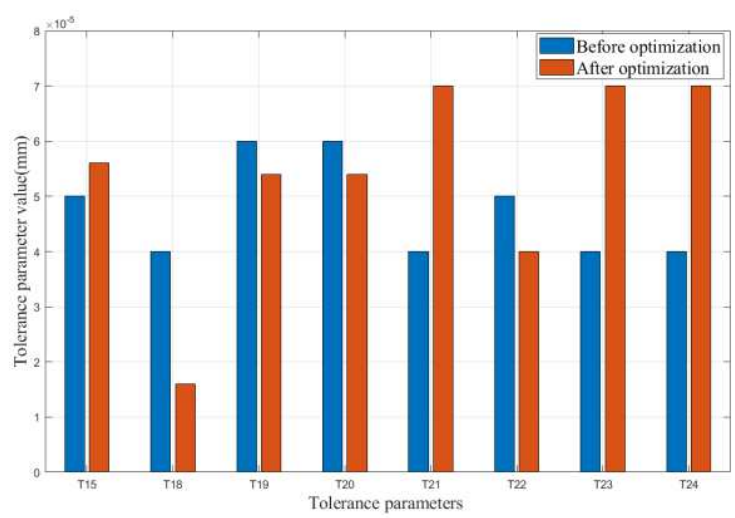

(b) Angular error

Figure 8 Comparison of tolerance parameters before and after optimization

\subsubsection{Experimental verification of tolerance parameter optimization allocation model}

This section will examine and demonstrate the optimal allocation results of the tolerance parameters of the key parts of the $\mathrm{CNC}$ internal cylindrical compound grinding machine. Based on the established grinder space motion error prediction model and the obtained optimized tolerance allocation plan, the space motion error of the whole machine will be predicted. Randomly select 2000 machining positions as sample points in the commonly used work space of the machine tool, and analyze the prediction results to verify the rationality of the tolerance optimization distribution results.

The components in the $\mathrm{X}, \mathrm{Y}$, and $\mathrm{Z}$ directions of the predicted spatial motion error corresponding to 2000 machining position points, as Figure 9 shown. The blue solid dot in the figure indicates that the predicted spatial motion error exceeds the maximum spatial motion error required by the machine tool design standard. The cyan-blue hollow dot indicates that the predicted value of the spatial motion error is lower than that required by the machine tool design standard. According to statistics, the number of qualified points for the prediction value of the spatial motion error in the X, Y, and Z directions are 1970, 1965, and 1957, respectively, the number of out-of-tolerance points are 30,35 , and 43, respectively, and the machining accuracy qualification rates are $98.5 \%, 98.25 \%$, and $97.85 \%$ respectively. It is obvious that the spatial motion error of the CNC internal cylindrical compound grinder after the optimization of the tolerance parameters meets the design requirements, which preliminarily verifies the rationality of the tolerance optimization distribution results and the correctness of the multi-objective optimization distribution model established in this paper. 


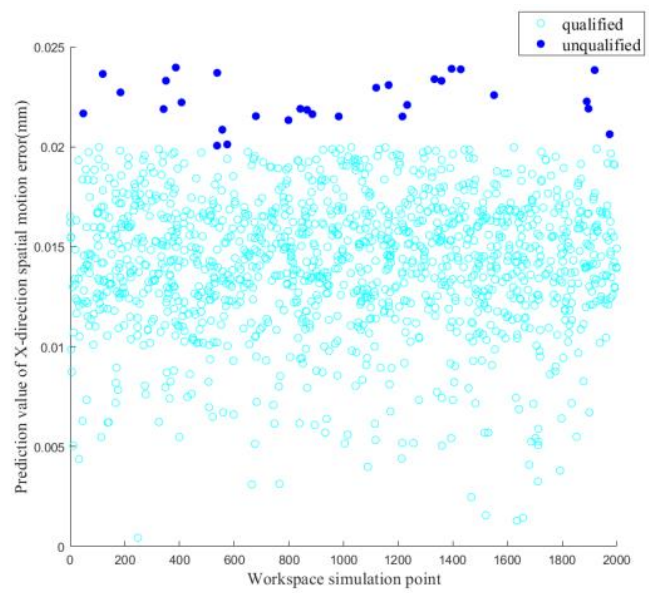

(a)X-direction

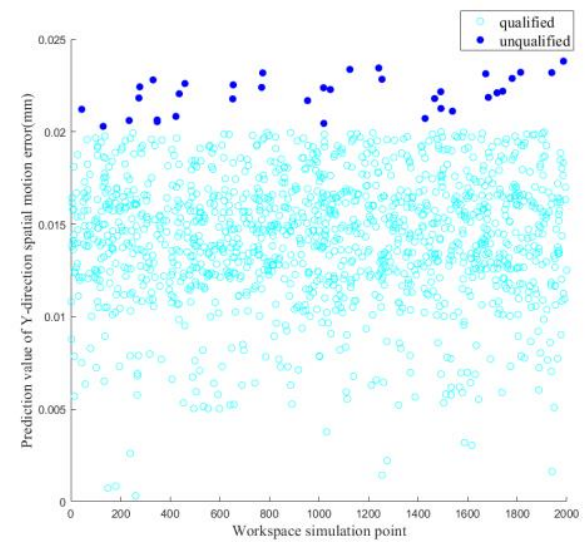

(b) Y-direction

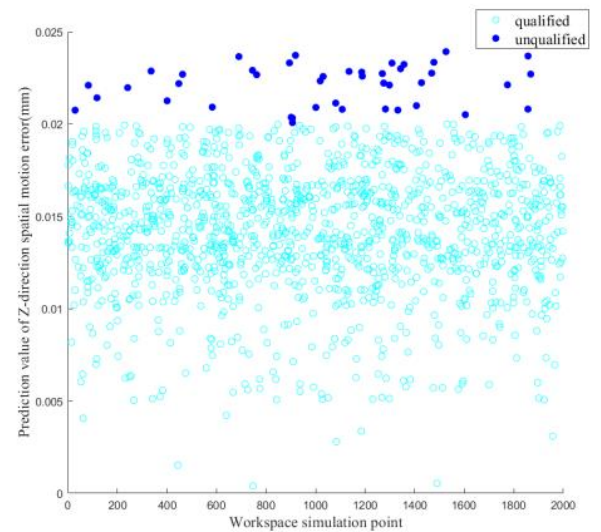

(c) Z-direction

Figure 9 Scatter plot of spatial motion error prediction value

\section{Conclusions}

This paper conducts an in-depth study on the machining error prediction of $\mathrm{CNC}$ internal circular compound machine tools and the optimal allocation of tolerance parameters of key parts of the machine tool, and proposes a new tolerance optimization allocation method to reduce the total manufacturing cost of the machine tool while meeting the machining accuracy requirements. The main contents include:

(1) Based on the Fourier series and the superposition function of the monotonic function and the Fourier series, the relationship model between the tolerances and geometric errors of the key parts of the machine tool is established; the machine tool is carried out based on the multi-body system theory. Simplify the analysis, derive the relationship model between the machine tool's spatial motion error and geometric error, and then obtain the relationship between the tolerance of the key parts of the machine tool and the machine's spatial motion error, and realize the machining error prediction.

(2) Based on the machining error prediction model of the CNC internal compound grinder, a sensitivity analysis model was constructed to obtain the sensitivity coefficients of various tolerance parameters.

(3) Established a multi-objective optimization model for the tolerance parameters of the key components of the CNC internal cylindrical compound grinder. Taking into account the position offset of the tool center point caused by its own gravity and the cutting force when the machine is working, ANSYS is used to analyze the offset of the tool center point. The final spatial motion error does not exceed its design standard and the tolerance value does not exceed 2 times its standard value as constraint conditions, the sensitivity coefficient of each tolerance parameter is used as the weighting factor, and the product of each tolerance parameter value and its corresponding weighting factor Maximizing the sum and minimizing the machining error of the whole machine are the design goals, and a multi-objective optimization distribution model of the tolerance parameters of the key parts of the CNC internal cylindrical compound grinder is established. The multi-objective optimization allocation model is solved based on the NSGA-II algorithm. The simulation results show that under the premise that the spatial motion error of the whole machine meets the requirements, most of the optimized tolerance parameters have been relaxed to varying degrees, which indirectly reduces the overall machine's The manufacturing cost preliminarily verifies the feasibility and effectiveness of the multi-objective optimization allocation model for the tolerance parameters of the key parts of the CNC internal cylindrical compound grinder proposed in this paper.

\section{Declaration}




\section{Acknowledgements}

Not applicable.

\section{Funding}

Supported by National Natural Science Foundation of China (Grant No. 51775010 and 51705011), Science and Technology Major Projects of High-end CNC Machine Tools and Basic Manufacturing Equipment of China (No.2019ZX04006-001).

\section{Availability of data and materials}

The datasets supporting the conclusions of this article are included within the article.

\section{Authors' contributions}

The author' contributions are as follows: Jin-Wei Fan was in charge of the whole trial; Ben-tian Xie wrote the manuscript; Qin-Zhi Zhao and Jun-Jian Wang assisted with sampling and laboratory analyses.

\section{Competing interests}

The authors declare no competing financial interests.

\section{Consent for publication}

Not applicable

\section{Ethics approval and consent to participate}

Not applicable

\section{References}

[1] Zeng S. Research on the development and trend of domestic CNC machine tools and technology [J]. Internal Combustion Engines and Accessories, 2021(04): 95-96. (in Chinese)

[2] Cheng Q, Qi B, Liu Z, Zhang C, Xue D (2019) An accuracy degradation analysis of ball screw mechanism considering time-varying motion and loading working conditions. Mech MachTheory 134:1-23.

[3] Liu Q.The development history and future trends of CNC machine tools[J].China Mechanical Engineering,2021,32(07):757-770. (in Chinese)

[4] JAYAPRAKASH G, SIVAKUMAR K, THILAK M. FEA compliant parametric tolerance allocation of mechanical assembly using neural network and differential evolution algorithm[J]. International Journal of Computer Integrated Manufacturing. 2012, 25(7): 636-654.

[5] Sivakumar K, Balamurugan C, Ramabalan S (2011) Concurrent multi-objective tolerance allocation of mechanical assemblies considering alternative manufacturing process selection. Int $\mathbf{J}$ Adv Manuf Technol 53:711-732.

[6] GEETHA K, RAVINDRAN D, KUMAR M S. Concurrent tolerance allocation and scheduling for complex assemblies [J]. Robotics and Computer-Integrated Manufacturing.2015, 35: 84-95.

[7] CAI L G, ZHANG Z L, CHENG Q, et al. An approach to optimize the machining accuracy retainability of multi-axis $\mathrm{NC}$ machine tool based on robust design [J]. Precision Engineering.2016, 43: 370-386.

[8] ZHAO Y M, LIU D S, WEN Z G. Optimization design method of product general tolerance system [J]. The International Journal of Advanced Manufacturing Technology. 2014, 70:363-374.

[9] LIU S G, JIN Q, DONG Y H, et al. A closed-form method for statistical tolerance allocation considering quality loss and different kinds of manufacturing cost functions [J]. The International Journal of Advanced Manufacturing Technology. 2017, 93: 2801-2811.

[10] Liu H B, Liu J H, He Y X et al. Tolerance multi-objective optimization design based on niche particle swarm optimization[J].Computer Integrated Manufacturing System, 2015, 21(3):585-592. (in Chinese)

[11] Shi X L, Xu X M, Su LL, et al. Multi-objective optimization of aerospace product assembly tolerances based on NSGA- II algorithm[J]. Shanghai Aerospace (Chinese and English), 2020, 37(03): 121-125+146. (in Chinese)

[12] ZHANG Z L, CAI L G, CHENG Q, et al. A geometric error budget method to improve machining accuracy reliability of multi-axis machine tools [J]. Journal of Intelligent Manufacturing. 2019, 30: 495-519.

[13] Qin Y C. Research on Intelligent Design Method of Tolerance Specification Based on Ontology[D]. Huazhong University of Science and Technology, 2017(in Chinese)

[14] Jiang P (2019) Research on modeling and compensation of the position independent geometric errors in five-axis denture machining center [D]. Hefei University of Technology, Hefei

[15] Wu C, Fan J, Wang Q, et al. Machining accuracy improvement of non-orthogonal five-axis machine tools by a new iterative compensation methodology based on the relative motion constraint equation [J]. International Journal of Machine Tools and Manufacture, 2018, 124:80-98.

[16] EKINCI T O, MAYER J R R. Relationships between straightness and angular kinematic errors in machines [J]. International Journal of Machine Tools and Manufacture. 2007, 47:1997-2004.

[17] Tao H H,Fan J W,Wu C J.A tolerance-based method for predicting geometric errors of machine tool guideways[J].Engineering Science and Technology,2020,52(04):235-242. (in Chinese)

[18] Chase K, Greenwood WK (1990) Least cost tolerance allocation for mechanical assemblies with automated process selection. Manuf Rev 3:49-59.

[19] Shao J W, Ma Y L, Zhang Z J, et al. Research on tolerance allocation based on a new tolerance cost model [J]. Machine Design. 2003, 20(2): 59-62. (in Chinese)

[20] 20. CHENG K M, TSAI J C. Optimal statistical tolerance allocation for reciprocal exponential cost-tolerance function $[\mathrm{J}]$. Proceedings of the Institution of Mechanical Engineers, Part B:Journal of Engineering Manufacture. 2013, 227(5): 650-656.

[21] Zhang P P,Zheng B M,Wang S Y.Research on Multi-Object Robust Equipment Layout Based on Improved NSGA-II[J].Modular Machine Tool and Automatic Manufacturing Technology,2018(12):125-128. (in Chinese)

\section{Biographical notes}

Jin-Wei Fan, born in 1965, Graduated from Tianjin University in 1996 with a Ph.D., director of the Institute of Precision CNC Machining Technology, Beijing University of Technology. His main research direction is CNC technology, precision machining and servo control technology, China.

Tel: +86 18813053935; E-mail: jwfan@ bjut.edu.cn 
Ben-Tian Xie, born in 1997, Graduate student from the Institute of Precision CNC Machining Technology, Beijing University of Technology, his main research direction is CNC technology, precision machining and servo control technology, China.

E-mail:1540397074@qq.com

Qin-Zhi Zhaog, born in 1963, China National Machine Tool Quality Supervision Testing Center, China.

E-mail:zqz629@126.com

Jun-Jian Wang, born in 1965, China National Machine Tool Quality Supervision Testing Center, China.

E-mail: wangjunjian@jcs.gt.cn

\section{Appendix 1}

$\left\{\begin{array}{l}\delta_{x}(x)=\mathrm{k} * x+T 1 \sin \left(\frac{2 \pi x}{\lambda}\right) \\ \left.\left.\delta_{y}(x)=\frac{T 2\left(\sin \left(\frac{2 \pi x+\pi H}{\lambda}\right)+\sin \left(\frac{2 \pi x-\pi H}{\lambda}\right)\right)}{2}\right)+\sin \left(\frac{2 \pi x-\pi H}{\lambda}\right)\right) \\ \left.\left.\delta_{z}(x)=\frac{T 3\left(\sin \left(\frac{2 \pi x+\pi H}{\lambda}\right)\right.}{2}\right)+\sin \left(\frac{2 \pi x-\pi H}{\lambda}\right)\right) \\ \left.\left.\varepsilon_{x}(x)=\frac{T 4\left(\sin \left(\frac{2 \pi x+\pi H}{\lambda}\right)\right.}{2 B}\right)-\sin \left(\frac{2 \pi x-\pi H}{\lambda}\right)\right) \\ \varepsilon_{y}(x)=\frac{T 3\left(\sin \left(\frac{2 \pi x+\pi H}{\lambda}\right)\right.}{H} \\ \varepsilon_{z}(x)=\frac{T 2\left(\sin \left(\frac{2 \pi x+\pi H}{\lambda}\right) \sin \left(\frac{2 \pi x-\pi H}{\lambda}\right)\right)}{H}\end{array}\right.$

$$
\left\{\begin{array}{l}
\delta_{x}(B)=T 16 \sin \theta_{B} \\
\delta_{y}(B)=T 16 \sin \theta_{B} \\
\delta_{z}(B)=R \frac{T 16}{L_{B}} \sin \theta_{B} \\
\varepsilon_{x}(B)=\frac{T 16}{L_{B}} \sin \theta_{B} \\
\varepsilon_{y}(B)=\frac{T 16}{L_{B}} \sin \theta_{B} \\
\varepsilon_{z}(B)=T 18 \sin \theta_{B}
\end{array}\right.
$$

\section{Appendix 2}

$\int E_{x}=-\delta_{x}\left(x_{1}\right)+(z+R \cos B) \varepsilon_{y}\left(x_{1}\right)+\left(q_{6 y}-q_{1 y}+\varepsilon_{z}\left(x_{1}\right)+(R \cos B+z) \varepsilon_{x_{1} B}+\delta_{x}\left(x_{2}\right)-\right.$ $R \cos B \varepsilon_{y}\left(x_{2}\right)+\left(1-q_{6 y}\right) \varepsilon_{z}\left(x_{2}\right)-q_{7 y} \varepsilon_{x_{2} B}+R \cos B \varepsilon_{x_{2} z}-\delta_{x}(A)+\left(q_{6 y} \sin A-q_{3 y} \sin A-\right.$ $\left.q_{1 y} \sin A+q_{5 y} \sin A-z \cos A-R \cos B \cos A-q_{2 y} \sin A+q_{7 y} \sin A\right) \varepsilon_{y}(A)+\left(-q_{6 y} \cos A+\right.$ $\left.q_{3 y} \cos A+q_{1 y} \cos A+z \sin A-R \cos B \sin A+q_{2 y} \cos A-q_{7 y} \cos A\right) \varepsilon_{z}(A)+(z+\cos B) \varepsilon_{z A}+$ $\cos \mathrm{B} \delta_{\mathrm{x}}(\mathrm{B})-\delta_{\mathrm{z}}(\mathrm{B})-q_{7 y} \sin B \varepsilon_{x}(B)+R \cos B \cos B \varepsilon_{y}(B)+\cos B \varepsilon_{z}(B)-\delta_{x}(z)+$ $(z+R \cos B) \varepsilon_{y}(z)+\left(q_{6 y}-q_{1 y}+q_{5 y}+1\right) \varepsilon_{z}(z)$

$E_{y}=\cos A \delta_{y}\left(x_{1}\right)-\sin A \delta_{z}\left(x_{1}\right)+\left(z \cos A-q_{2 y} \sin A+q_{1 y} \sin A-q_{5 y} \sin A\right) \varepsilon_{x}\left(x_{1}\right)+\left(x_{2} \sin A-x_{1} \sin A+\right.$ $\left.q_{1 x}\right) \varepsilon_{y}\left(x_{1}\right)+\left(x_{2} \cos A+q_{1 x} \cos A\right) \varepsilon_{z}\left(x_{1}\right)+\left(x_{2} \sin A+q_{1 x} \sin A\right) \varepsilon_{x x_{z}}-\cos A \delta_{y}\left(x_{2}\right)+$

$\left(R \sin B \sin A-q_{6 y} \cos A-q_{7 y} \sin B\right) \varepsilon_{x}\left(x_{2}\right)-\cos A \varepsilon_{z}\left(x_{2}\right)-\cos A \varepsilon_{x_{2} B}+\left(x_{2} \sin A+q_{1 x} \sin A\right) \varepsilon_{x_{2} z}-$ $\delta_{y}(A)+\left(-q_{6 y} \sin A+z \cos A+q_{3 y} \sin A-\sin A \varepsilon_{y}\left(x_{2}\right)-q_{2 y} \sin A-q_{5 y} \sin A\right) \varepsilon_{x}(A)+$

$\left(x_{1}-x_{2}+q_{3 x}-q_{1 x}\right) \varepsilon_{z}(A)-\sin B \sin A \delta_{x}(B)-\cos A \delta_{y}(B)+\cos B \sin A \delta_{z}(B)-\cos A \varepsilon_{x_{2} B}+$ $\cos A \delta_{y}(z)-\sin A \delta_{z}(z)-\left(q_{7 y} \sin B \sin A+q_{1 y} \cos A-q_{5 y} \cos A-z \cos A+R \sin B \sin A-q_{6 y} \cos A\right) \varepsilon_{x}(z)+$ $\left(x_{2} \sin A+q_{1 x} \sin A\right) \varepsilon_{y}(z)+\sin A \delta_{z}\left(x_{2}\right)+\left(x_{2} \cos A+\cos A+q_{1 x} \cos A\right) \varepsilon_{z}(z)$

$E_{z}=-\cos A \delta_{z}\left(x_{1}\right)+\left(-R \sin A \sin B+q_{6 y} \cos A+q_{7 y} \cos A\right) \varepsilon_{x}\left(x_{1}\right)+\left(x_{1} \cos A-\cos A+q_{1 x} \cos A-\right.$ $\left.x_{2} \cos A\right) \varepsilon_{y}\left(x_{1}\right)-\left(q_{1 x} \sin A+x_{2} \sin A+x_{1} \sin A\right) \varepsilon_{z}\left(x_{1}\right)+\left(q_{1 x} \cos A+x_{2} \cos A-\cos A\right) \varepsilon_{x_{x z}}-\sin A \delta_{y}\left(x_{2}\right)-$ $\cos A \delta_{z}\left(x_{2}\right)+q_{6 y} \sin A \varepsilon_{x}\left(x_{2}\right)+\cos A \varepsilon_{y}\left(x_{2}\right)+\left(q_{1 x} \cos A-x_{2} \cos A-\cos A\right) \varepsilon_{x_{2 z}}-\delta_{z}(A)+$ $\left(-q_{3 y} \cos A-R \sin B \sin A+q_{6 y} \sin A\right) \varepsilon_{x}(A)+\left(x_{2} \sin A-q_{3 x} \sin A+\sin A+q_{1 x} \sin A\right) \varepsilon_{z A}+$ $\left(-q_{1 x}+x_{2}-x_{1}+q_{3 x}+1\right) \varepsilon_{y}(A)+\left(q_{3 x} \cos A-x_{2} \cos A-x_{1} \cos A-\cos A\right) \varepsilon_{z A}-\cos B \cos A \delta_{z}(B)+$ $\left(q_{7 y} \sin B \sin A+R \sin B \sin A\right) \varepsilon_{z B}+\left(q_{1 y} \sin A-q_{6 y} \sin A-q_{5 y} \sin A\right) \varepsilon_{x}(z)+$ $\left(q_{1 x} \cos A+x_{2} \cos A\right) \varepsilon_{y}(z)+\left(x_{2} \sin A-q_{1 x} \sin A\right) \varepsilon_{z}(z)+\cos A \delta_{z}(z)$ 